\title{
Measuring Diversity on a Low-Altitude UAV in a Ground-to-Air Wireless 802.11 Mesh Network
}

\author{
H. T. Kung, Chit-Kwan Lin, Tsung-Han Lin, Stephen J. Tarsa, Dario Vlah \\ Harvard University \\ Cambridge, MA
}

\begin{abstract}
We consider the problem of mitigating a highly varying wireless channel between a transmitting ground node and receivers on a small, low-altitude unmanned aerial vehicle (UAV) in a 802.11 wireless mesh network. One approach is to use multiple transmitter and receiver nodes that exploit the channel's spatial/temporal diversity and that cooperate to improve overall packet reception. We present a series of measurement results from a real-world testbed that characterize the resulting wireless channel. We show that the correlation between receiver nodes on the airplane is poor at small time scales so receiver diversity can be exploited. Our measurements suggest that using several receiver nodes simultaneously can boost packet delivery rates substantially. Lastly, we show that similar results apply to transmitter selection diversity as well.
\end{abstract}

\section{INTRODUCTION}

Small, low-altitude unmanned aerial vehicles (UAVs) have reached a development point where they are useful in many real-world applications, both military and civilian, such as surveillance, reconnaissance, photography, communications, environmental and traffic monitoring, etc. At the same time, researchers are investigating new UAV-related problems such as autonomous control, swarming, mesh networking, airborne computing, etc. These topics have a common need for an ever increasing amount of wireless communications and networking, making this an important support area in UAV research. In particular, commercial-off-the-shelf technologies such as IEEE 802.11 wireless LAN have made it practical to build low-cost aerial wireless mesh networks [1], [2], [3].

However, a key challenge to deployment is achieving good link qualities. Rapid changes in signal strength due to UAV banking maneuvers and outages dictated by environmental shadowing or flight paths translate into a highly varying and lossy wireless channel between ground transmitters and UAV receivers. This is of particular concern to fixed-wing aircraft, which are attractive due to their efficiency and relatively high mobility but have physical flight path induced complications.

While one could use better or more powerful radios, similar issues of high link loss and variation could still arise at larger distances. Since it is well-known that node position and orientation can greatly impact wireless link quality [4], [5], we consider an alternative approach where multiple receiver nodes on-board a UAV cooperate to boost packet delivery rates from a ground transmitter by exploiting the spatial and temporal diversity of the wireless channel. This scheme also easily extends to multiple ground transmitters by means of

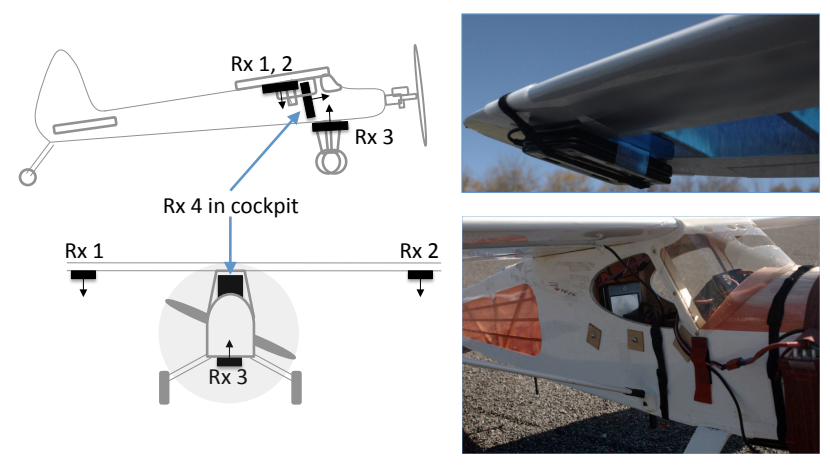

Fig. 1: Mounting positions of receiver MID nodes on the UAV; arrows show the direction in which the screen of each device faced. The photos show the MID mounted on the starboard wing and inside the cockpit.

selection, whereby we periodically choose the active ground transmitter.

We present a series of measurement results on a real-world UAV testbed to (1) characterize the wireless channel between multiple ground transmitter and multiple UAV receiver nodes and (2) determine the gain achievable via diversity. Our data show that UAV receiver nodes exhibit poor packet reception correlation at short time scales. By exploiting this property we demonstrate that multiple transmitters and receivers can be used to improve packet delivery rates significantly.

\section{Field EXPERIMENT SETUP}

We collected ground-to-air packet traces by recording individual packet transmissions and receptions originating from a fixed ground transmitter node to four UAV-mounted receiver nodes, respectively. (We have applied a similar approach in the air-to-ground direction, and will report those results in a future publication). In this section, we describe our experimental setup for collecting these traces.

The UAV we used had a 110" wingspan, a radio-transparent fuselage, and aluminum landing gear and wing struts. Its electric engine was powered by batteries that permit a maximum flight time of 20 minutes. The UAV also had an autopilot that follows a flight path defined by way points. With low wind, it achieved high positional accuracy across laps-e.g., we have found positional deviation with respect to a fixed way point to be no more than $13 \mathrm{~m}$ when the plane was level, and no more than $25 \mathrm{~m}$ when the plane was banking through a turn. This 
permitted repeatable data collection over multiple laps within a flight and over multiple flights. During a flight, we logged altitude, airspeed, GPS latitude/longitude, and GPS time every $250 \mathrm{~ms}$.

At an altitude of $75 \mathrm{~m}$, our UAV followed a cyclical, dumbbell-shaped flight path that passed beyond the radio range at the extremes (Figures 2a-2d). Each lap ran clockwise and, at an airspeed of $20 \mathrm{~m} / \mathrm{s}$, lasted $\sim 90$ s. Cruising time for each flight was $\sim 15$ minutes, meaning the UAV covered ten laps per flight. We collected packet traces over four flights in total. These flights were conducted at a property surrounded by woods and farmland, free of 802.11 radio traffic.

Our wireless mesh network nodes were Mobile Internet Devices (MIDs) that feature an $800 \mathrm{MHz}$ x86-compatible Intel Atom processor, a Marvell SD8686 802.11b/g internal SDIO radio, an internal omnidirectional antenna and Ubuntu Linux 8.04. The antenna orientation was determined by the direction at which the MID screen faces. One transmitter MID was placed amid tall grass and brush, elevated $20 \mathrm{~cm}$ from the ground, with its screen facing skyward. Its location is shown as a square in Figures 2 and 4. Four receiver MID nodes (Rx1 to $\mathrm{Rx} 4$ ) were mounted on the UAV as shown in Figure 1. $\mathrm{Rx} 1$ and $\mathrm{Rx} 2$ were mounted with screens facing the ground, one on the underside of each wing tip. Rx3, with its screen facing skyward, was mounted between the landing gear and $\mathrm{Rx} 4$, with its screen facing the bow, was mounted inside the cockpit. The direction of mounting is significant since measurements we took in an anechoic chamber show that the MID's antenna pattern is asymmetric (Figure 3). Thus, our mounting configuration provides diversity in antenna patterns and offers different shadowing profiles from the landing gear, engine and batteries.

We placed all the 802.11 nodes in ad-hoc mode using the 1 Mbps modulation. To collect traces, our transmitter broadcast 1420-byte UDP packets, with unique sequence numbers, at 80 $\mathrm{pkt} / \mathrm{s}$. The receiver nodes on the UAV ran a client that logged the sequence number and timestamp of each received packet.

i

\section{EXPERIMENT RESULTS}

In this section we present experiment results and discuss their significance. We begin with a look at basic single-receiver properties of the collected data. Next, we examine the gain of receive diversity, due to multiple receivers, and discuss its sources. Lastly, we present measurements of transmit diversity, due to multiple transmitters.

\section{A. Baseline Single-receiver Performance}

To gain a qualitative understanding of reception behavior in our particular flight pattern we analyzed our collected traces as follows. For each receiver, we first divided its packet traces into 20-packet windows, forming a windowed trace for each flight, and then calculated the packet loss rate over each window. This window size captures the loss rate over a reasonably short distance: 20 packets correspond to $250 \mathrm{~ms}$ of

\begin{tabular}{ccccc} 
Flight \# & $\mathrm{Rx} 1$ & $\mathrm{Rx} 2$ & $\mathrm{Rx} 3$ & $\mathrm{Rx} 4$ \\
\hline 1 & 0.41 & 0.44 & 0.20 & 0.35 \\
2 & 0.34 & 0.37 & 0.23 & 0.32 \\
3 & 0.30 & 0.38 & 0.17 & 0.28 \\
4 & 0.35 & 0.40 & 0.24 & 0.27
\end{tabular}

TABLE I: Overall packet delivery rates achieved by the four receivers during four separate flights.

\begin{tabular}{cccc} 
Flight \# & Full Loss & Intermediate & No Loss \\
\hline 1 & $47.3 \%$ & $18.6 \%$ & $34.1 \%$ \\
2 & $54.8 \%$ & $18.3 \%$ & $26.9 \%$ \\
3 & $55.1 \%$ & $16.1 \%$ & $28.8 \%$ \\
4 & $52.5 \%$ & $18.3 \%$ & $29.2 \%$
\end{tabular}

TABLE II: Percent of flight traces with full loss, intermediate signal, and no loss, determined using a 20-packet window.

flight time, or roughly $5 \mathrm{~m}$ at a nominal airspeed of $20 \mathrm{~m} / \mathrm{s}$. Figures $2 \mathrm{a}-2 \mathrm{~d}$ plot the location of each window from all four flights and categorize each window as no loss (green; 0\% loss), full loss (red; $100 \%$ loss) or intermediate loss (yellow; all others). Table I contains the overall packet delivery rates for each receiver and each flight.

At first glance the overall packet loss rates seem quite high. However, it is useful to separate the regions where the UAV is out of range, since those regions could make the loss rates seem arbitrarily high. Hence, Table II shows the makeup of each flight across the 3 loss categories. We can see that after factoring out the full loss portions, the four flights have 47.5- 52.7\% of their traces consist of both the no loss and intermediate loss regions. Suppose that these regions constitute $50 \%$ of these traces. Then the packet delivery rates excluding the full loss portions of the traces should be approximately twice of those reported in Table I.

There are two major implications of these findings. First, the periodic full- and intermediate-loss regions resulting from the circulatory flight pattern will be an important factor in UAV network protocol design, especially with use of legacy protocols such as Transmission Control Protocol (TCP) [6], [7] which are sensitive to packet loss patterns. Secondly, the significant presence of intermediate loss regions (16.1-18.6\% in Table II) indicates that we could obtain substantial gains through the use of receiver diversity, provided that the losses at different receivers are generally not correlated. We explore this possibility in the next section.

\section{B. Diversity of Multiple Receivers}

Figures $2 \mathrm{a}-2 \mathrm{~d}$ show that the loss regions are different in size and location across the four receivers. This is further confirmed in Figures $2 \mathrm{e}-2 \mathrm{~h}$, which takes a packet trace from a single representative flight (Flight 2) and simply plots the location of each received packet over the course of the flight for each receiver node. For instance, Rx1 receives packets in the north- 


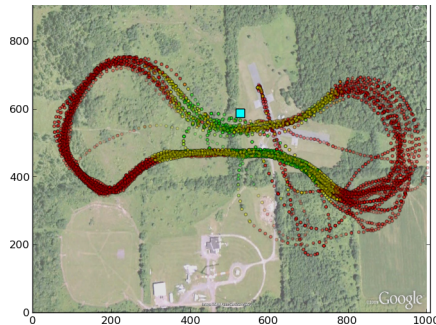

(a) $\mathrm{Rx} 1$

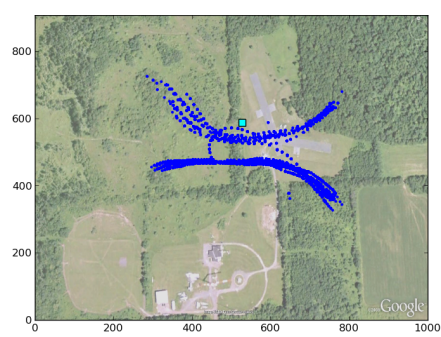

(e) $\operatorname{Rx} 1$

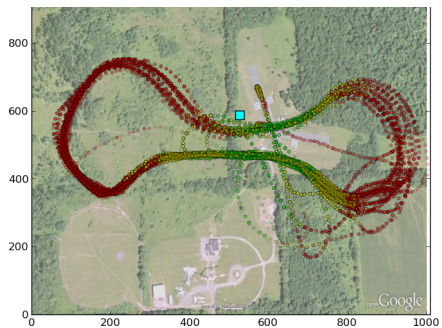

(b) $\mathrm{Rx} 2$

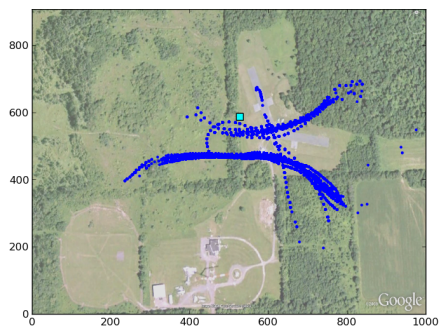

(f) $\mathrm{Rx} 2$

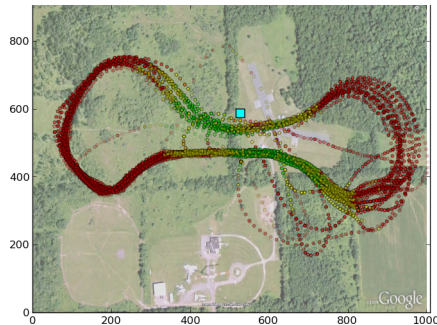

(c) $\mathrm{Rx} 3$

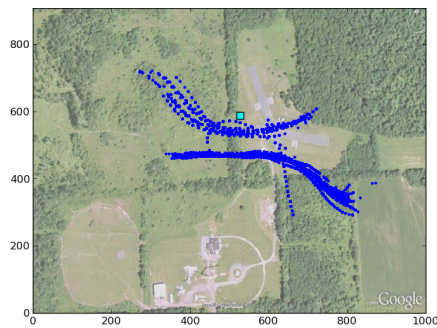

(g) $\mathrm{Rx} 3$

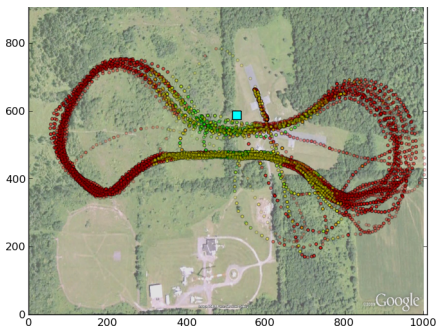

(d) $\mathrm{Rx} 4$

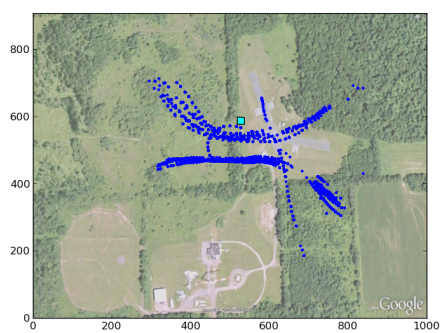

(h) $\mathrm{Rx} 4$

Fig. 2: Packet loss and reception over the flight path. The top of the page is the northerly direction and the square indicates the location of the ground transmitter node. (a)-(d): Packet loss rates over all four flights, per receiver. Each point denotes a 20 packet window over which the loss rate is calculated; green is no loss, red is full, and yellow is intermediate. Note the autopilot system gives a stable trajectory over multiple laps and flights (points falling outside the dumbbell-shaped path are from human piloting during take-off and landing). (e)-(h): Location of each packet reception event, per receiver, during a single flight (Flight 2).
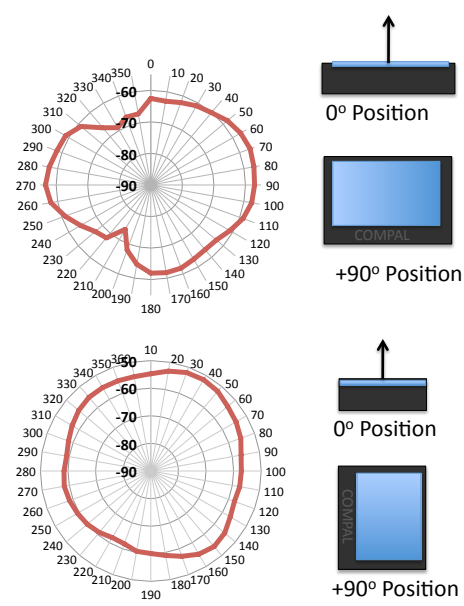

Fig. 3: RSSI in $\mathrm{dB}$ where the MID receiver is rotated about one axis, as measured in an anechoic chamber. The transmitter (not shown) is positioned at the $+90^{\circ}$ orientation. Lower: the $0^{\circ}-180^{\circ}$ measurements indicate the relative signal strength the wing-mounted receivers would experience when passing directly over a ground transmitter; the $180^{\circ}-360^{\circ}$ measurements for the undercarriagemounted MID; and the $90^{\circ}-270^{\circ}$ measurements for the cockpitmounted MID. Upper: rotations around the long axis of the device occur during UAV banking for the wing and bottom MID, and UAV changing direction for the cockpit MID.

west quadrant (i.e., on approach towards the ground node), but $\mathrm{Rx} 2$ does not until it is almost flying over the ground node.

The above behaviors led us to examine the reception correlation amongst pairs of receiver nodes over the course of

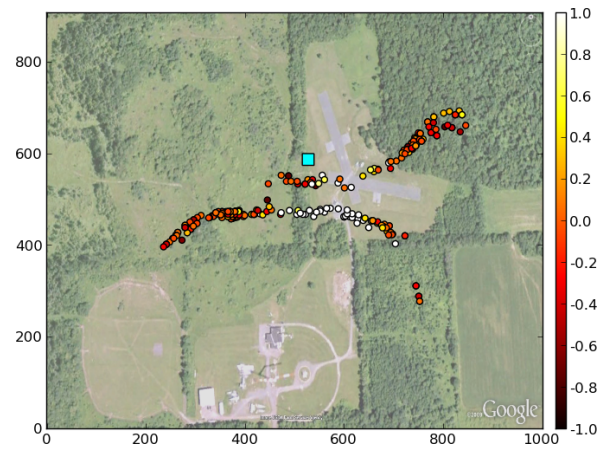

Fig. 4: Reception event correlation coefficient $(\rho)$ between a representative pair of receivers over Flight 2 . The color of each point is the value of the correlation coefficient over a 20 packet window. The top of the figure is the northerly direction and the square indicates the location of the ground transmitter node.

a flight (Flight 2). To do so, we first synchronize the packet traces of two receiver nodes, generate the windowed traces for each as before, and then calculate the correlation coefficient

$$
\rho=\frac{E\left[\left(X-\mu_{X}\right)\left(Y-\mu_{Y}\right)\right]}{\sigma_{X} \sigma_{Y}}
$$

of the packet reception pattern in pairs of windows $X$ and $Y$ corresponding to the same location along the flight path for the two receivers. Note that the correlation coefficient is not defined for any window pair in which at least one window has either no loss or full loss; we ignore such window pairs as a result. We performed this operation for all pairs of receivers.

Figure 4 plots the results for each receiver pair assuming 


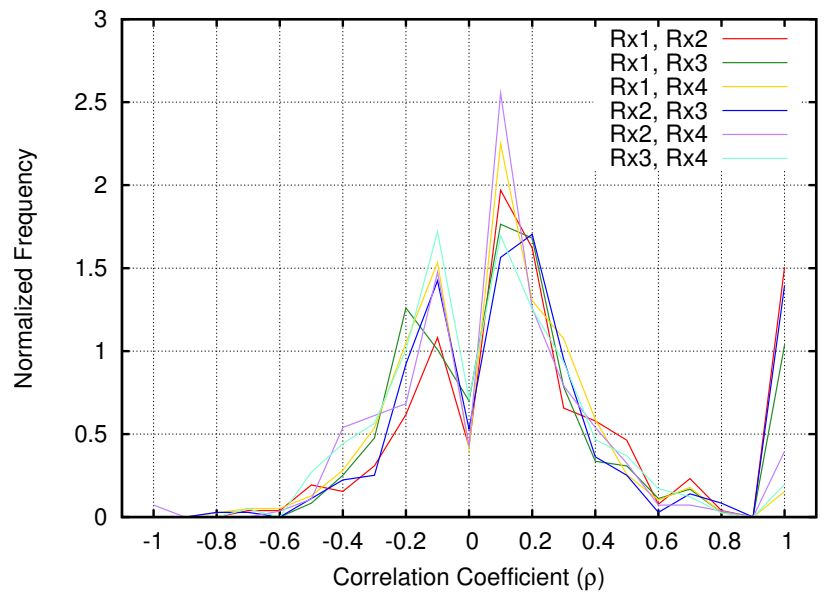

Fig. 5: Normalized histogram of the correlation coefficient $(\rho)$ calculated over 20-packet windows for each receiver pair during Flight 2 .

20-packet windows. The color of each data point indicates the $\rho$ value for that location. Receivers mounted externally $(\mathrm{R} \times 1, \mathrm{R} \times 2$, and $\mathrm{Rx} 3)$ were well-correlated when the UAV passed close to the ground node, but were poorly correlated further away. This is also demonstrated in Figure 5, which is a normalized histogram of the $\rho$ values from Flight 2 for each receiver pair. While all pairs have significant mass in $\rho=[-0.2,0.2]$, indicating a general lack of correlation over most of the flight path, only externally-mounted node pairs show an additional large peak at $\rho=1.0$, a region of high correlation where the UAV passes near the ground node. In contrast, pairs $(\mathrm{Rx} 1, \mathrm{Rx} 4)$ and $(\mathrm{Rx} 2, \mathrm{Rx} 4)$, which correspond to the wing/cockpit node pairs, lack this peak entirely and are actually the most uncorrelated nodes since they have the overall largest peaks at $\rho=0.1$. This disparity is due to $\mathrm{Rx} 4$ being mounted in the cockpit and having an antenna orientation significantly different from that of the wing-mounted nodes (see Figure 3). Taken together, these results indicate that receivers are uncorrelated over large portions of the flight path and suggest that employing multiple receiver nodes to cooperatively boost receiver diversity can help improve packet delivery rates on-board the UAV.

To determine the gain due to receiver diversity, we combine the packet receptions at the four receivers into a combined trace, where a packet is marked as received if it was received by at least one individual receiver. Table III shows the diversity performance in terms of the overall delivery rates of combined traces for each flight, alongside the best single receiver's delivery rate. We can see that diversity brings about an additional gain of roughly $25 \%$.

\section{Diversity at Various Time Scales}

We next discuss the availability of diversity gain at various time scales in our experiments. First, on large time scales of, say, one flight lap, the reception traces are clearly correlated, resulting from all receiver nodes experiencing nearly identical

\begin{tabular}{l|cccc} 
& Flight 1 & Flight 2 & Flight 3 & Flight 4 \\
\hline $\begin{array}{l}\text { Best single } \\
\text { receiver }\end{array}$ & $\mathrm{Rx} 2$ & $\mathrm{Rx} 2$ & $\mathrm{Rx} 2$ & $\mathrm{R} \times 2$ \\
$\begin{array}{l}\text { Best single } \\
\text { delivery rate }\end{array}$ & 0.44 & 0.37 & 0.38 & 0.40 \\
$\begin{array}{l}\text { Combined } \\
\text { delivery rate }\end{array}$ & 0.55 & 0.49 & 0.46 & 0.51 \\
$\begin{array}{l}\text { Percent } \\
\text { improvement }\end{array}$ & $25 \%$ & $32 \%$ & $21 \%$ & $28 \%$
\end{tabular}

TABLE III: Combined packet delivery rates achieved by the four receivers during four separate flights, and the resulting receiver diversity gains.

path loss-as the mobile node moves far enough out of range of the transmitter, the loss of signal strength due to distance cannot be overcome by small differences between the receivers. Thus, receiver diversity might not help much here since all receivers undergo the same outage. Similarly, when the receivers are in close proximity of the transmitter, the loss rate is low enough that diversity cannot significantly improve it.

It is more interesting to examine diversity behavior at medium time scales, e.g., 1/8 of a lap. At this scale, the distance may not change enough to cause a major shift in path loss, and thus other effects become more pronounced. Namely, links may be shadowed diversely by various obstructions, such as the airplane as it banks, or even external objects like trees.

Lastly, on small time scales, where path loss does not change significantly due to either changing distance or shadowing, links may still undergo independent rapid variations due to fast fading. Fast fading occurs when multipath signals combine with a varying phase difference, e.g., due to Doppler effect or varying path lengths. The salient question is whether fast fading is present in our experiment environment, and if so, to what extent it occurs independently at each receiver.

We evaluate diversity at different time scales in two ways. First, we compute the correlation of all receiver pairs over increasing time spans. Secondly, we compare the diversity gain in our traces to that of synthetic traces generated using a Bernoulli process.

1) Correlation vs. time scale: We compute the correlation between two receiver traces as follows. For a window size of $w$ packets, we draw 1000 pairs of $w$-packet trace segments starting at randomly chosen points in the traces, and compute the average correlation coefficient, but not counting segments where all or none packets were lost. We repeat the computation for window sizes ranging from 1 to 10000 packets, or 135 seconds. Figure 6 shows the resulting correlations.

Two features stand out in the plot. First, there is a clear difference between the correlation of different receiver pairs. The pair with most correlation, $\mathrm{Rx} 1$ and $\mathrm{Rx} 2$, are the two receivers mounted on the underside of the wings, corroborating the finding from Section III-B. At the same time, the pair with least correlation are $\mathrm{Rx} 3$ and $\mathrm{Rx} 4$, which differ not only 


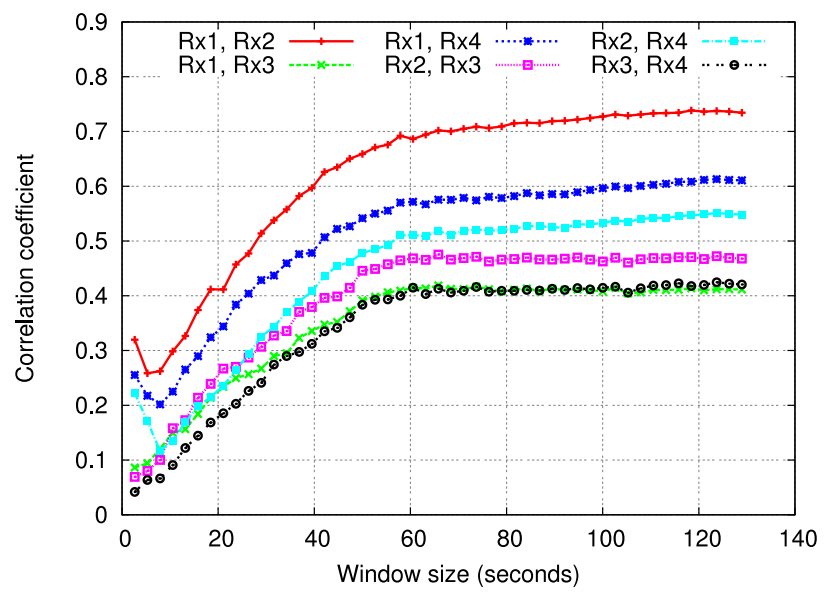

Fig. 6: Correlation behavior at different time scales. Shown are correlation plots for 6 possible receiver pairs.

in mounting orientation but also in the nature of line-of-sight obstruction; Rx3 is mounted on the UAV's bottom while $\mathrm{Rx} 4$ is inside the cockpit area.

The second main feature of the plot is the apparent sharp increase in correlation at window sizes of up to about 60s. This time scale is large enough that it covers both in- and outof-range regions of the flight path, where all receivers start to experience the same large-scale path-loss effects. Below this window size, however, correlation decreases, indicating a lack of dependence due to fading. Finally, as window size decreases further, the correlation increases again due to increasing fraction of windows with all or no packet losses, skewing the average correlation coefficient.

2) Comparison with Bernoulli traces: We now look for further evidence of independent packet loss behavior at short time scales. In particular, we compare the collected traces to synthetic Bernoulli traces constructed as follows. Let us divide a collected trace into $N$ segments of length $w$ packets. For each segment $i$, we compute its packet delivery rate, $p_{i}$. For each $i$, we construct a synthetic packet loss segment by performing $w$ Bernoulli trials with parameter $p_{i}$. Finally, we concatenate the $N$ segments, repeat the process for all receivers' traces, and merge the traces into a combined trace as described at end of Section III-B.

The diversity performance of synthetic traces should correspond closely to that of a system where receivers experience only the independent Gaussian noise. In Figure 7 we present the diversity performance of synthetic traces for a range of window sizes $w$, and compare to performance of the system in our experiment. We can see that, indeed, the performances match very closely at short time scales, and begin to diverge at window sizes similar to those of increasing correlation in Figure 6. This seems to indicate that fading has little effect in our environment, and that packet loss behavior is largely independent at short time scales.

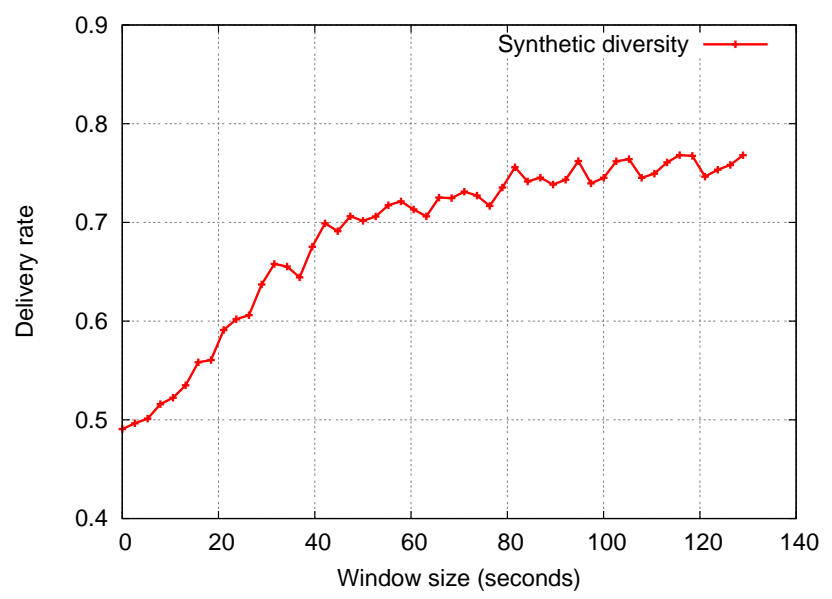

Fig. 7: Diversity performance in terms of delivery rate of a set of synthetic traces created using a Bernoulli process based on data from Flight 2 . In contrast, the diversity performance of real traces from the same flight is 0.49 , as Table III indicates.

\section{Multiple-Transmitter Diversity Using Selection}

In addition to the single-transmitter flights discussed in the previous sections, we conducted one flight with 3 ground transmitters which took turns broadcasting one full-size UDP packet at a time. We controlled the timing of these transmissions precisely using a wired control network. Thus, we obtained the channel qualities for 12 links ( 3 transmitters $\times 4$ receivers) but at $1 / 3$ the resolution, as the transmissions were interleaved.

Let us assume that transmitters obtain channel quality feedback every $w$ packets, so that every $w$ packets our system can select the best transmitting node. Thus, we can calculate the transmitter selection diversity gain by dividing the traces into size- $w$ windows, and construct a combined trace by taking the best performing ones. Additionally, for each window, we can calculate the performance either to a single receiver or multiple receivers using receiver diversity, thus obtaining performance under several combinations of transmitter and receiver diversity. We present the resulting delivery rates in Table IV, calculated for $w=27$, or roughly 1 second; we discuss the choice of this value below. We can see that transmit diversity gives us another significant increase in delivery rate, independent of whether receive diversity is used.

Unlike receiver diversity, transmitter selection diversity requires periodic channel quality feedback. A good choice for the feedback rate would introduce minimal overhead while still providing up-to-date channel quality information. To check the effect of this parameter in our experiment scenario, we recomputed transmit diversity gain from Table IV using a range of values for $w$; the results appear in Table V. We can see that most of the gain is already available at rates around $1 \mathrm{~s}$. 


\begin{tabular}{cccc} 
& Transmission pattern & Delivery rate & Percent improvement \\
\hline$\# 1$ & Tx2 to Rx2 & 0.41 & \\
$\# 2$ & all to Rx2 & 0.46 & $12 \%$ over \#1 \\
$\# 3$ & Tx2 to all & 0.51 & $24 \%$ over \#1 \\
$\# 4$ & all to all & 0.57 & $12 \%$ over \#3, 24\% over \#1
\end{tabular}

TABLE IV: Packet delivery rates achieved by 1) single transmitter to single receiver, 2) 3 transmitters to single receiver, 3) single transmitter to 4 receivers, and 4) 3 transmitters to 4 receivers. In single transmitter or receiver cases, we used the best performing node.

\begin{tabular}{l|ccccccc}
$\begin{array}{l}\text { Selection } \\
\text { window } \\
\text { (packets) }\end{array}$ & 1 & 10 & 27 & 50 & 100 & 500 & 1000 \\
\hline Delivery rate & 0.57 & 0.57 & 0.57 & 0.56 & 0.56 & 0.54 & 0.53
\end{tabular}

TABLE V: Packet delivery rates for increasing selection feedback intervals, in the 3 -to-4 transmission scenario. The 27 -packet interval corresponds to $1 \mathrm{~s}$.

\section{CONCLusions}

In this paper we presented results of an experimental study of ground-to-air UAV links. We found that the links are intermediate in their qualities for a significant amount of time, owing to the UAV moving in and out of range frequently; this might be a property specific to fixed-wing craft, but is important nonetheless as those make up the most efficient class of fliers. As a result, network layer protocols will need to be adapted to cope with this situation, or to work on top of link layer mechanisms which use, e.g., coding or retransmissions to mask the loss [7].

At small time scales of up to 1000 packets, or about 10 seconds, we found that the packet losses seem to be almost uncorrelated, giving diversity performance very similar to that of synthetic traces generated using Bernoulli trials. This seems to indicate that the effect of fast fading is not significant in our environment in any direction, in the sense that it does not result in either better or worse diversity performance than that of memoryless losses.

The diversity gain we observed with four receiver nodes consisted of an average $25 \%$ increase in delivery rate, and an additional $12 \%$ with three transmitter nodes using selection diversity. On one side, a question arises whether this is sufficient to warrant the addition of extra receivers on a UAV; in the event that the multiple receivers are already present in the UAV application, using them for diversity reception would provide a clear and inexpensive benefit. Finally, on the transmit side, using multiple transmitters introduces protocol complications related to feedback, synchronization, packet retransmissions, etc. However, as has been shown in previous work [8], many of these issues can be addressed using network coding techniques which reduce the need for frequent feedback.

\section{ACKNOWLEDGEMENTS}

This material is based on research sponsored by Air Force Research Laboratory under agreement numbers FA8750-09-
2-0180 and FA8750-10-2-0180. The U.S. Government is authorized to reproduce and distribute reprints for Governmental purposes notwithstanding any copyright notation thereon. The views and conclusions contained herein are those of the authors and should not be interpreted as necessarily representing the official policies or endorsements, either expressed or implied, of Air Force Research Laboratory or the U.S. Government.

\section{REFERENCES}

[1] T. Brown, B. Argrow, C. Dixon, and S. Doshi, "Ad Hoc UAV Ground Network (AUGNet)," in AIAA 3rd "Unmanned Unlimited" Technical Conference, 2004.

[2] A. Ryan, X. Xiao, S. Rathinam, J. Tisdale, M. Zennaro, D. Caveney, R. Sengupta, and J. K. Hedrick, "A modular software infrastructure for distributed control of collaborating uavs," in Proc. AIAA Conf. on Guidance, Navigation and Control, 2006.

[3] D. Hague, H. T. Kung, and B. W. Suter, "Field experimentation of cotsbased uav networking," in MILCOM, 2006.

[4] D. Aguayo, J. Bicket, S. Biswas, G. Judd, and R. Morris, "Link-level measurements from an 802.11b mesh network," in SIGCOMM, 2004.

[5] J. Bicket, D. Aguayo, S. Biswas, and R. Morris, "Architecture and evaluation of an unplanned 802.11b mesh network," in MobiCom, 2005.

[6] J. Postel, "Transmission control protocol," RFC 793, Sep. 1981.

[7] H. T. Kung, C.-K. Lin, T.-H. Lin, S. J. Tarsa, and D. Vlah, "A locationdependent runs-and-gaps model for predicting TCP performance over a UAV wireless channel," in MILCOM 2010 (to appear), San Jose, CA, USA, 2010.

[8] _ , "Flowcode: Multi-site data exchange over wireless ad-hoc networks using network coding," in MILCOM, 2009. 